\title{
O direito ao aborto no Brasil e a implicação da Atenção Primária à Saúde
}

\section{The right to abortion in Brazil and the role of Primary Health Care \\ El derecho al aborto en Brasil y el rol de la Atención Primaria de Salud}

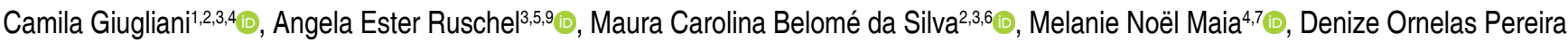 \\ Salvador de Oliveira ${ }^{4,8}$ (D)
}

${ }^{1}$ Departamento de Medicina Social, Universidade Federal do Rio Grande do Sul (UFRGS). Porto Alegre, RS, Brasil.

2 Programa de Pós-Graduação em Epidemiologia, Universidade Federal do Rio Grande do Sul (UFRGS). Porto Alegre, RS, Brasil.

${ }^{3}$ Fórum Aborto Legal do Rio Grande do Sul. Porto Alegre, RS, Brasil.

${ }^{4}$ Grupo de Trabalho Mulheres na Medicina de Família e Comunidade, Sociedade Brasileira de Medicina de Família e Comunidade (SBMFC). Rio de Janeiro, RJ, Brasil.

${ }^{5}$ Hospital Materno-Infantil Presidente Vargas, Secretaria Municipal de Saúde de Porto Alegre. Porto Alegre, RS, Brasil.

${ }^{6}$ Departamento de Ações em Saúde, Seção de Saúde das Mulheres, Secretaria Estadual de Saúde do Rio Grande do Sul. Porto Alegre, RS, Brasil.

${ }^{7}$ Clínica da Família Rinaldo De Lamare, Secretaria Municipal de Saúde do Rio de Janeiro. Rio de Janeiro, RJ, Brasil.

${ }^{8}$ Programa de Residência em Medicina de Família e Comunidade, Secretaria de Saúde de São Bernardo do Campo. São Bernardo do Campo, SP, Brasil.

${ }^{9}$ Programa de Pós-Graduação em Saúde Coletiva da Universidade Federal do Rio Grande do Sul (UFRGS). Porto Alegre, RS, Brasil.

\section{Resumo}

Apesar dos importantes progressos ocorridos no âmbito da saúde pública no Brasil, as mortes maternas por abortos inseguros representam um desafio persistente. Por tratar-se de condição prevalente na população, a mulher que busca o serviço de saúde por questões relacionadas ao aborto deve encontrar no serviço de atenção primária uma porta de entrada segura e um espaço de diálogo aberto. Considerando que o contexto legal do Brasil é um dos mais restritivos do mundo em relação ao aborto, este texto apresenta ideias de como o(a) médico(a) de família e comunidade pode atuar frente a uma situação de gravidez indesejada, baseado na experiência de outros países, considerando os atributos da atenção primária e o código de ética médica. Por fim, a experiência do Fórum Aborto Legal RS é relatada, no intuito de compartilhar ações concretas visando à qualificação do atendimento às mulheres que recorrem a um aborto permitido por lei. Conclui-se que, no nosso meio, um primeiro passo para avançar no direito ao aborto é fazer cumprir a lei que garante a sua realização em situações específicas e que a atuação dos(as) profissionais da atenção primária na redução de danos parece ser uma estratégia chave para evitar abortos inseguros e, por consequência, reduzir a mortalidade materna.

Palavras-chave: Aborto Induzido; Aborto Legal; Atenção Primária à Saúde; Medicina de Família e Comunidade

Como citar: Giugliani C, Ruschel AE, Silva MCB, Maia MN, Oliveira DOPS. O direito ao aborto no Brasil e a implicação da Atenção Primária à Saúde. Rev Bras Med Fam Comunidade. 2019;14(41):1791. http://dx.doi.org/10.5712/rbmfc14(41)1791

Autor correspondente: Camila Giugliani. E-mail: giugli@hotmail.com Fonte de financiamento: declaram não haver.

Parecer CEP: não se aplica.

Procedência e revisão por pares: revisado por pares.

Recebido em: 29/06/2018. Aprovado em: 19/12/2018. 


\begin{abstract}
Despite important progress in public health in Brazil, maternal deaths from unsafe abortion remain a continuous challenge. Because it is a prevailing condition in the population, a woman who seeks health care for abortion-related issues must find in the primary care facility a safe doorway and an open space for dialogue. Considering that Brazil's legal context is one of the most restrictive in the world in relation to abortion, this text presents ideas on how the family physician can act in dealing with a situation of unwanted pregnancy, based on the experience of other countries and considering the primary care attributes and the medical ethics code. Finally, the experience of the Legal Abortion Forum in Rio Grande do Sul is reported, in order to share concrete actions aimed at the qualification of care for women who seek an abortion that is granted by law. In conclusion, in our country, a first step to acquire more significant progress on the right to abortion is to enforce the law that guarantees its fulfillment in specific situations. Moreover, the engagement of primary care professionals in harm reduction seems to be a key strategy to prevent unsafe abortion and, consequently, to reduce maternal mortality.
\end{abstract}

Keywords: Abortion, Induced; Abortion, Legal; Primary Health Care; Family Practice

\title{
Resumen
}

A pesar de los importantes progresos ocurridos en el ámbito de la salud pública en Brasil, las muertes maternas por abortos inseguros representan un desafío persistente. Por tratarse de condición prevalente en la población, la mujer que busca el servicio de salud por cuestiones relacionadas al aborto debe encontrar en el servicio de atención primaria una puerta de entrada segura y un espacio de diálogo abierto. Considerando que el contexto legal de Brasil es uno de los más restrictivos del mundo en relación al aborto, este texto presenta ideas de cómo el (la) médico (a) de familia y comunidad puede actuar frente a una situación de embarazo no deseado, basado en la experiencia de otros países, considerando los atributos de la atención primaria y el código de ética médica. Por último, se comparte la experiencia del Foro Aborto Legal de Rio Grande do Sul, con el fin de proponer acciones concretas para la calificación de la atención a las mujeres que recurren a un aborto permitido por ley. Se concluye que, en nuestro contexto, un primer paso para avanzar en el derecho al aborto es hacer cumplir la ley que garantiza su realización en situaciones específicas y que la actuación de los profesionales de la atención primaria en la reducción de riesgos y daños parece ser una estrategia clave para evitar abortos inseguros y, por consiguiente, reducir la mortalidad materna.

Palabras clave: Aborto Inducido; Aborto Legal; Atención Primaria de Salud; Medicina Familiar y Comunitaria

\section{O aborto: uma questão de gênero e um problema de Saúde Pública}

As discussões sobre o aborto são extensas e antigas. Existem controvérsias que variam de intensidade sobre se e quando o aborto deve ser permitido e quais as situações que o justificam ou sobre quando se inicia a vida. Valores éticos como poder, igualdade, dignidade, direitos, liberdade, justiça, fé, moral e autonomia se atravessam nessa trama de discussão. ${ }^{1}$

O termo aborto comporta diferentes significados, que variam conforme o contexto cultural, político ou legal. Na área da saúde, abortamento é a interrupção da gestação (espontânea ou voluntária) até 20 ou 22 semanas com o embrião ou feto pesando menos de $500 \mathrm{~g}$; já o aborto, é o produto do abortamento. Contudo, na prática, os dois termos são utilizados como sinônimos. ${ }^{2}$

No campo da saúde coletiva, o abortamento é classificado em três grandes tipos: espontâneo ou induzido; legal ou ilegal; e seguro ou inseguro. ${ }^{2} \mathrm{O}$ entendimento desses conceitos é importante para a prática cotidiana dos serviços de saúde nos diferentes níveis de atenção. No entanto, há uma grande diferença no acesso aos cuidados de saúde, bem como no atendimento recebido, para as mulheres que buscam tratamento diante de um aborto espontâneo e para aquelas que procuram os serviços em decorrência de complicações após um aborto provocado, ou ainda para aquelas que buscam o procedimento do aborto nos casos previstos em lei. No caso do aborto induzido, pode haver situações que vão desde o julgamento moral até a denúncia policial pelo(a) profissional de saúde. Em se tratando do aborto legal, além do julgamento moral, há a questão do difícil acesso devido a uma rede de serviços pequena, despreparada e pouco articulada. 
Estima-se que, a cada ano, cerca de 230 mil mulheres internem pelo Sistema Único de Saúde (SUS) em decorrência de abortos inseguros. ${ }^{2}$ Além disso, o aborto tem sido uma das principais causas de óbito materno no Brasil nos últimos anos. ${ }^{3} \mathrm{O}$ aborto é, portanto, uma importante questão de saúde pública. Renomados pesquisadores reconhecem que, apesar dos importantes progressos ocorridos no Brasil, as mortes maternas por abortos inseguros representam um desafio persistente. ${ }^{4}$

A Pesquisa Nacional de Aborto de 2016 mostra que o aborto inseguro é frequente entre as brasileiras. O estudo aponta que cerca de $13 \%$ das 2.002 entrevistadas, na faixa etária dos 18 aos 39 anos, todas alfabetizadas e residentes em áreas urbanas, já fizeram ao menos um aborto na vida. Aplicando-se a taxa de aborto encontrada na pesquisa, o número de mulheres que teriam feito o aborto em 2015 seria de aproximadamente 503 mil. ${ }^{5}$ Essa pesquisa também mostrou que mulheres de todas as classes sociais, níveis educacionais, idades e religiões recorrem ao aborto, ainda que as taxas de realização variem significativamente segundo grupos, sendo mais altas em mulheres pretas, pardas, amarelas e indígenas, com menor escolaridade e menor renda. Todavia, no contexto de ilegalidade, clandestinidade e insegurança, são mulheres pobres, negras e jovens as que possuem os maiores riscos de adoecimento e óbito. ${ }^{5}$ Outro fator relevante para a saúde pública é que muitas mulheres que teriam o direito de acessar os serviços de saúde para realizarem a interrupção da gravidez prevista em lei acabam por optar por meios inseguros, justamente por desconhecerem seus direitos ou por não serem bem acolhidas e bem atendidas nos serviços do SUS.

Entre 2013 e 2015, foram realizados apenas 2.442 abortos legais em decorrência de estupro no Brasil. ${ }^{6}$ No entanto, segundo o Anuário Brasileiro de Segurança Pública de 2016, calcula-se a frequência de cerca de um estupro a cada 11 minutos no país. Isso significa que, embora o número de ocorrências em 2015 tenha sido de 45.460, estima-se que o número real fique entre 129,9 mil e 454,6 mil estupros, devido à subnotificação. ${ }^{7}$ Considerando que $5 \%$ a $7 \%$ das mulheres vítimas de estupro podem engravidar, percebe-se que o número de abortos legais realizados no território nacional está bem abaixo do esperado. ${ }^{8}$

A escassez de serviços para o atendimento das mulheres que recorrem ao aborto previsto em lei e a dificuldade no tratamento das complicações decorrentes de um aborto inseguro devem ser entendidas como uma violação dos direitos sexuais e reprodutivos. Esse cenário também deve ser compreendido à luz das questões de gênero predominantes em nosso contexto social.

Um exemplo clássico da influência das relações de gênero e de seus reflexos sociais foi a incorporação das políticas de saúde voltadas para a mulher nas primeiras décadas do século XX no Brasil. Os programas materno-infantis, elaborados nas décadas de 30, 50 e 70, traduziam uma visão restrita sobre a mulher, baseada em sua especificidade biológica e no seu papel social de mãe, responsável pela criação, educação e pelo cuidado com a saúde dos filhos e dos demais familiares. ${ }^{9}$ Embora esse cenário tenha se modificado razoavelmente, percebe-se até hoje a ênfase dada ao parto e ao nascimento, em contraposição ao aborto, tópico historicamente negligenciado.

Adicionalmente, também é necessário entender a questão da violência de gênero como um grave problema social. O estupro, assim como as demais violências de gênero, não trata de sexo, de afetividade ou de intimidade. Trata, sim, de uma relação de poder, em que as mulheres devem assumir um estado de subalternação sem serem sujeitas dos próprios desejos ou donas dos direitos sobre o seu corpo. ${ }^{10}$ 
As redes de atenção do SUS são imprescindíveis para a implementação de ações que garantam às mulheres o acesso aos direitos sexuais e reprodutivos, ao aborto legal e ao atendimento seguro nos casos de complicações decorrentes de abortos inseguros. Nesse sentido, a atuação da Atenção Primária à Saúde (APS) precisa ser entendida para além dos métodos contraceptivos e da atenção ao pré-natal. $A$ APS deve funcionar como um espaço de diálogo aberto e como uma porta de entrada segura para a mulher que busca o serviço de saúde por questões relacionadas ao aborto. Diante do cenário de desigualdade, iniquidade e negligência do nosso país, é preciso que a saúde pública - começando pela APS - assuma o seu protagonismo para a garantia do acesso aos direitos sexuais e reprodutivos.

\section{O aborto legal no Brasil}

No Brasil, o aborto é considerado crime; entretanto, o Código Penal Brasileiro, ${ }^{11}$ em seu artigo 128 , não pune a realização do aborto nas seguintes situações: gravidez que coloque em risco a vida da mulher e gravidez resultante de estupro. Em 2012, o Supremo Tribunal Federal (STF) incluiu nesse rol o direito ao aborto nos casos de anencefalia. Apesar dessa antiga previsão jurídica, foi apenas em 1989 que o primeiro serviço de aborto legal foi criado no Brasil, no Hospital Jabaquara, em São Paulo, proporcionando atendimento a mulheres vítimas de violência sexual. Esse serviço é considerado pioneiro, na América Latina, em oferecer assistência gratuita para realização do aborto legal, destacando-se também por ter se antecipado em uma década em relação ao Ministério da Saúde (MS), que estabeleceu sua primeira norma técnica sobre o tema apenas em 1999.12

A publicação da Norma Técnica sobre Prevenção e Tratamento dos Agravos Resultantes da Violência Sexual contra Mulheres e Adolescentes, ${ }^{12}$ que previu a realização do abortamento legal no âmbito do SUS, demarcou a estruturação desses serviços no Brasil. Além disso, em 2013, o MS publicou a portaria que definia o funcionamento dos serviços de Atenção Integral às Pessoas em Situação de Violência Sexual, que também incluía a regulação dos serviços de aborto legal. ${ }^{13}$

Entende-se "Serviço de Aborto Legal" como um local de referência para a realização do procedimento de aborto permitido por lei no SUS. Prevê a constituição de uma equipe multidisciplinar composta por ginecologistas-obstetras, psicólogas(os), assistentes sociais e enfermeiras(os) que atuam no atendimento à violência sexual e também na realização do aborto. A referida Norma Técnica passou por duas atualizações, em 2005 e 2012. A principal mudança em relação à Norma original, inserida em 2005, foi o reconhecimento de que a narrativa da mulher é suficiente para averiguar a presença de violência sexual, ou seja, exclui-se a obrigatoriedade de apresentação do boletim de ocorrência policial. ${ }^{14}$

Apesar desse avanço na proposição de uma política pública direcionada à saúde das mulheres vítimas de violência sexual, o acesso ao aborto ainda não é uma garantia para as mulheres brasileiras. Em 2005, havia apenas 56 hospitais cadastrados com equipes especializadas para a interrupção da gestação em caso de violência sexual. No entanto, apenas 37 deles estavam capacitados para realizar o procedimento e 5 nunca tinham feito qualquer aborto legal. Além disso, em seis estados brasileiros, ainda não havia nenhum serviço organizado, e a maioria dos atendimentos estava centralizada na Região Sudeste, que acabava recebendo mulheres de todas as demais regiões. ${ }^{15}$ 
Infelizmente, quase uma década depois, outra pesquisa demonstrou que não houve mudança significativa nesse cenário. Dos 68 serviços existentes entre 2013 e 2015, apenas 37 haviam realizado interrupções de gravidez decorrente de estupro. Novamente, constatou-se a concentração de serviços no Sudeste e a ausência de serviço ativo em sete estados. Algumas das instituições ainda exigiam boletim de ocorrência policial, laudo do IML ou alvará judicial para o procedimento. ${ }^{5}$

Pesquisas como essas demonstram que as mulheres que engravidam de um estupro podem ter seu direito violado por questões geográficas, institucionais ou de falta de informação, além de questões envolvendo a consciência dos profissionais que as atendem. A resistência dos médicos para a realização do procedimento é uma das dificuldades percebidas: segundo Faúndes et al., ${ }^{16}$ apesar de a maioria dos profissionais concordar com o direito da mulher de interromper uma gestação decorrente de estupro, apenas $50 \%$ demonstram disponibilidade para realizar o procedimento. A maioria das publicações nacionais diz respeito aos conhecimentos, atitudes e práticas de ginecologistas-obstetras sobre o aborto, não havendo estudos específicos sobre médicos de família e comunidade.

Recentemente, novos encaminhamentos legais têm trazido à tona a discussão sobre o aborto. O STF, em novembro de 2016, definiu que o aborto não deveria ser considerado crime no primeiro trimestre da gravidez, absolvendo cinco pessoas pertencentes à equipe médica de uma clínica clandestina de abortos no Rio de Janeiro. ${ }^{17}$ Em contrapartida, houve forte reação por parte da bancada evangélica no Congresso Nacional, por meio da Proposta de Emenda Constitucional 181 (PEC 181). O projeto inicial, que propunha alterar o artigo $7^{\circ}$ da Constituição Federal, permitindo a ampliação da licença-maternidade em caso do nascimento de bebês prematuros, foi transformado com inserções no texto constitucional. No inciso 3 do artigo $1^{\circ}$ da Constituição, que trata dos princípios fundamentais, a redação passaria a ser "a garantia da inviolabilidade da vida desde a concepção" e, no artigo $5^{\circ}$, acrescentou-se a mesma expressão à redação: "a inviolabilidade do direito à vida desde a concepção". ${ }^{18}$ Ressalte-se que a comissão foi basicamente formada por homens, e que, se aprovada, a proposta cercearia os direitos sexuais e reprodutivos e inviabilizaria o direito ao aborto nos termos que até então a legislação brasileira prevê.

\section{O aborto na prática da Atenção Primária}

Pelo exposto, o aborto parece ser um tema mais frequente na vida das mulheres brasileiras do que nos consultórios médicos. No país, aproximadamente $55 \%$ das gestações que chegam a termo não são planejadas, ${ }^{19}$ e a legislação restritiva vigente faz com que as mulheres enfrentem gestações indesejadas sem o aconselhamento adequado, e, muitas vezes, realizem abortos de maneira insegura, colocando em risco a própria vida. ${ }^{20}$

Considerando que a violência sexual é, infelizmente, tão frequente no Brasil, também são frequentes as gestações em sua decorrência, principal motivo para a realização de um aborto dentre os tipos previstos em lei. Dessa forma, por tratar-se de condição prevalente, a devida importância a esse problema deve ser dada no contexto da atenção primária.

Outros países trazem exemplos de como podemos abordar o problema da gravidez indesejada, mesmo em contextos nos quais o aborto é criminalizado. No Uruguai, por exemplo, antes da descriminalização do aborto, que ocorreu somente em 2012, foi implementada uma exitosa estratégia de redução de danos 
para prevenção de mortes maternas relacionadas ao aborto inseguro..$^{21,22} \mathrm{O}$ pilar central dessa estratégia era o contato com o serviço de saúde para aconselhamento antes e depois da realização da interrupção voluntária da gestação.

Dessa forma, todas as mulheres que demonstravam alguma incerteza em relação à decisão de manter uma gestação eram convidadas para atendimento em um serviço especializado em saúde sexual e reprodutiva, onde, no primeiro atendimento, eram realizadas as seguintes ações: confirmação de gestação viável e determinação da idade gestacional, apuração de situações em que o aborto seria permitido por lei, aconselhamento sobre outras possibilidades para o desfecho da gestação (incluindo a adoção) e fornecimento de informações sobre métodos seguros e inseguros de abortamento. Considerando a legislação restritiva vigente no Uruguai naquela época, os profissionais não estavam autorizados a praticar a interrupção da gravidez, nem a orientar onde obter o Misoprostol, porém, o aborto medicamentoso era apresentado como um método seguro utilizado em países onde o aborto é permitido por lei, e todas as informações científicas pertinentes eram fornecidas, como posologia, vias de administração, sintomatologia, efeitos colaterais e sinais de alarme.

O objetivo desse atendimento não era de interferir na decisão das mulheres, mas de proporcionar a elas, como cidadãs, um acolhimento adequado e o acesso às informações disponíveis para uma melhor tomada de decisão. Um objetivo secundário era o estabelecimento de uma relação de confiança com a equipe de saúde, minimizando os temores relacionados à ocorrência de denúncia às autoridades policiais ou outras formas de violência institucional. ${ }^{21}$

Na estratégia uruguaia, todas as mulheres, independentemente da decisão tomada, eram convidadas para uma consulta "pós", para cuidados pós-abortamento ou início do acompanhamento pré-natal. Para as mulheres que haviam optado por abortar, era avaliada a ocorrência de abortamento incompleto e realizado esvaziamento uterino quando necessário e, principalmente, era disponibilizado um método contraceptivo efetivo conforme a escolha da mulher. ${ }^{21}$

Esse modelo de cuidados propiciou uma redução significativa e sustentada da mortalidade materna relacionada ao abortamento inseguro no país, ${ }^{22}$ antes mesmo da descriminalização do aborto, que ocorreria anos mais tarde. Isso mostra que, mesmo em países com legislação restritiva como o nosso, há muito o que os profissionais de saúde podem fazer para prevenir mortes maternas por aborto inseguro. A adoção do modelo de redução de danos, inclusive, foi um passo importante para chegar à mudança legal no Uruguai. Por seu êxito, a experiência foi replicada em outros países da América Latina, como Peru ${ }^{23}$ e Argentina. ${ }^{24}$

As intervenções descritas na experiência uruguaia são simples e facilmente adaptáveis ao contexto da APS, onde devemos atuar em três frentes para a redução das mortes maternas relacionadas ao aborto inseguro: ${ }^{25}$

- prevenção primária: evitar a gestação indesejada a partir de ações de educação sexual e planejamento reprodutivo;

- prevenção secundária: atuar para que o abortamento que não se pode evitar seja realizado de forma segura; e

- prevenção terciária: tratar oportunamente as complicações do abortamento e fornecer contracepção pós-abortamento para prevenir a sua repetição. 
Dentre essas ações, cabe destacar o importantíssimo papel do(a) profissional da atenção primária em fornecer informação de qualidade para as mulheres, e também para os homens, no que diz respeito aos direitos sexuais e reprodutivos, atuando de forma contínua no seu empoderamento, para tomarem decisões informadas e buscarem a realização dos seus direitos.

No caso de uma gravidez indesejada, seja em situação de aborto previsto em lei ou não, o(a) médico(a) de família e comunidade (MFC) ou outro(a) profissional da APS tem uma grande oportunidade de ser efetivo na resolução de um problema grave. Considerando os atributos essenciais da APS, ${ }^{26}$ uma ampla gama de ações pode (e deve) ser disponibilizada:

- $\quad$ acolhimento adequado, com atitude empática, sem julgamento ou discriminação (acesso de primeiro contato);

- $\quad$ atendimento às necessidades da mulher em situação de gravidez indesejada, considerando o seu contexto de vida e buscando a perspectiva de outros profissionais da equipe (integralidade);

- continuidade do atendimento, conforme os acontecimentos, ajudando a mulher no seu planejamento reprodutivo, resolvendo intercorrências clínicas ou prestando cuidado pré-natal (longitudinalidade); e

- em caso de aborto legal, conhecer a organização da rede de atenção à saúde do seu município e orientar a mulher quanto aos serviços de referência para atendimento e realização dos procedimentos, ajudando-a nos encaminhamentos. Os procedimentos que devem ser realizados em caso de violência sexual são: anticoncepção de emergência, profilaxias para sífilis, clamídia, gonococo, hepatite B e HIV e, no caso de uma gravidez indesejada, a interrupção da gestação conforme desejo da mulher (coordenação do cuidado).

Dependendo da estrutura da unidade básica de saúde e da agilidade do encaminhamento, alguns desses procedimentos já podem ser feitos antes do encaminhamento para o serviço de referência. Nesse aspecto, é de fundamental importância informar à mulher que: ela tem direito a esses procedimentos, que o serviço deve acolhê-la sem discriminação e com respeito, e que não é necessário apresentar boletim de ocorrência policial para ser atendida; que existe uma norma técnica no Brasil, a Norma Técnica de Atenção Humanizada ao Abortamento, ${ }^{27}$ que respalda o cumprimento desses direitos; e que ela terá a garantia da continuidade do seu acompanhamento na atenção primária, já enfatizando a importância do planejamento reprodutivo presente e futuro, discutindo com ela sobre as opções de métodos anticonceptivos, destacando-se os de longa duração (como DIU, implante subcutâneo e métodos definitivos, conforme a preferência da mulher).

Outra questão importante que o(a) médico(a) de família deve saber é que toda mulher tem direito de gozar dos benefícios do progresso científico e de suas aplicações. Esse direito à informação é respaldado pela Organização Mundial de Saúde (OMS), com base em tratados internacionais de direitos humanos e declarações de consenso globais. ${ }^{28}$ Aliado ao respaldo conferido pelo Código de Ética Médica ${ }^{29}$ nesse quesito, aqui encontramos a base legal para oferecer informações que estão amplamente disponíveis, tanto em nível acadêmico quanto para o público geral, e que podem ser determinantes para evitar um aborto inseguro, a exemplo da experiência uruguaia. 
Para as mulheres que já tomaram a decisão de abortar, mesmo que de forma ilegal, e a compartilharam com o(a) seu(ua) médico(a) de família, muitas informações podem ser discutidas em consulta, com a intenção de ajudá-las a encontrar os caminhos mais seguros, incluindo: confirmação da idade gestacional e segurança dos diferentes métodos, uso correto dos medicamentos no caso de aborto medicamentoso, sinais de alerta que indiquem a necessidade de procurar um serviço de saúde - todas essas informações encontram-se detalhadamente no Guia da OMS "Abortamento seguro: orientação técnica e de políticas para sistemas de saúde", ${ }^{30}$ com acesso livre na internet.

Sabe-se que o aborto é um procedimento muito seguro, se realizado com técnica correta e condições adequadas de higiene, atentando-se para a idade gestacional, cujo avanço implica em aumento de risco. Antes das 12 semanas, especialmente antes das 9 semanas, o risco de complicações de um aborto é inclusive menor que o risco envolvido em uma gestação de risco habitual levada a termo. ${ }^{30}$ Compartilhar informação de qualidade, disponível amplamente, é um ato de respeito e de cuidado com as pessoas que atendemos, que não infringe nenhuma lei ou norma vigente no nosso país; pelo contrário, está em conformidade com o código ético da profissão médica, conforme detalhado abaixo. É fundamental ter uma postura acolhedora e de não julgamento, pois a criminalização do aborto e o estigma envolvido fazem com que muitas mulheres deixem de dividir sua situação com os(as) profissionais de saúde, mesmo em casos permitidos pela lei. É interessante notar que, em países onde o aborto é legalizado, como em grande parte dos EUA, as mulheres preferem realizar o procedimento no serviço de APS, com o(a) seu(sua) médico(a) de família. ${ }^{31}$

\section{O aborto e a Ética Profissional}

A temática do aborto aflora questões morais e éticas na sociedade, nos profissionais de saúde e nas escolas médicas. O Código de Ética Médica ${ }^{29}$ determina que é vedado ao médico descumprir a legislação específica nos casos de abortamento, e o Código Penal ${ }^{11}$ prevê pena de reclusão de um a quatro anos por "provocar aborto com o consentimento da gestante", salvo nas situações de risco de vida materno ou de gravidez resultante de estupro. Não existe no Código Penal qualquer artigo relacionado ao aconselhamento para redução dos riscos associados ao aborto inseguro, não caracterizando esta ação, portanto, como infração à legislação vigente.

O Código de Ética Médica, por sua vez, coloca como princípios fundamentais ao exercício da profissão: agir em benefício à saúde do ser humano com o máximo de zelo e o máximo de sua capacidade profissional, usar o melhor do progresso científico em benefício do seu paciente, guardar absoluto respeito pelo ser humano e atuar sempre em seu benefício. Dentre as responsabilidades profissionais, é vedado ao médico "permitir que interesses pecuniários, políticos, religiosos, (...) interfiram na escolha dos melhores meios de prevenção, diagnóstico ou tratamento disponíveis e cientificamente reconhecidos no interesse da saúde do paciente ou da sociedade".

No capítulo referente aos direitos humanos, postula-se que é vedado ao médico "deixar de garantir ao paciente o exercício do direito de decidir livremente sobre sua pessoa ou seu bem-estar, bem como exercer sua autoridade para limitá-lo", e no tocante à relação com pacientes e familiares, é vedado ao médico "Deixar de usar todos os meios disponíveis de diagnóstico e tratamento, cientificamente reconhecidos e a seu alcance, em favor do paciente". 
É frequente no discurso de profissionais de saúde a alegação de que questões éticas os impedem de atuar no aconselhamento de mulheres que manifestem o desejo de abortar. Entretanto, ferimos nossa ética profissional quando omitimos informações relevantes e cientificamente embasadas que podem salvar a vida de uma mulher que está decidida a abortar e que, para isso, vai recorrer a métodos muitas vezes perigosos. Da mesma forma, constitui quebra de sigilo médico a denúncia às autoridades policiais de mulheres que provocaram aborto, apesar de esta ainda ser uma prática frequente em hospitais brasileiros.

\section{O aborto e a Educação Médica}

Um estudo realizado com acadêmicos de medicina de três universidades paulistas a respeito dos conhecimentos sobre aborto medicamentoso mostrou dados preocupantes. ${ }^{32}$ Apesar de todos os estudantes do último ano já terem ouvido a respeito do uso do Misoprostol para indução do aborto, e cerca de $90 \%$ relatarem que foram orientados sobre o modo de uso, as suas respostas a questões mais específicas mostraram que somente $20 \%$ apresentavam conhecimento satisfatório.

Em relação à Residência Médica, mesmo em países em que o aborto é legalizado, como em grande parte dos EUA, um estudo da Society of Teachers of Family Medicine (STFM) demonstrou que os programas de residência tinham limitações importantes em prover treinamento abrangente e recomendava ações para qualificar os currículos no tópico do aborto. ${ }^{33} \mathrm{Em} \mathrm{2013}$, os achados de Herbitter et al. ${ }^{34}$ também demonstraram, por meio de inquéritos com professores e preceptores de MFC, que nos EUA e Canadá, onde o aborto também é legalizado, o treinamento abrangente não estava amplamente disponível para os residentes, mesmo sendo reconhecido como parte integrante do cuidado centrado no paciente.

Outro estudo realizado nos EUA chamou atenção para a necessidade de fornecer treinamento sobre aborto de maneira fundamentada nos valores da medicina de família, como a continuidade do cuidado e os aspectos psicossociais da medicina. Ao observar o valor de prestar cuidados aos pacientes em um momento potencialmente difícil, idealmente em um ambiente de atenção primária, os residentes demonstraram como o treinamento para o aborto é coerente com a missão da MFC de fornecer continuidade de cuidados ao longo do ciclo de vida. ${ }^{35}$

A palavra aborto só aparece uma vez no Currículo Baseado em Competências da SBMFC, dentro das competências essenciais de urgência e emergência: "Reconhece, diagnostica, trata e refere adequadamente emergências obstétricas, como: aborto em curso, trabalho de parto, doença hipertensiva específica da gestação (DHEG) e pielonefrite." Os conhecimentos, habilidades e atitudes relacionados ao manejo das situações de gravidez indesejada ficam ocultos na competência essencial "Maneja apropriadamente os problemas mais frequentes e relevantes na saúde da mulher". ${ }^{36}$

Considerando que o aborto é um assunto tabu no nosso país, é esperado que haja resistência à discussão no âmbito das instituições de ensino, mesmo quando demonstrado que há uma forte demanda de estudantes de medicina e médicos(as) residentes em torno do esclarecimento de questões éticas, legais, sobre redução de danos e prevenção de agravos decorrentes de abortos inseguros, como demonstrado pelo alto interesse nas atividades relacionadas à temática realizadas em congressos regionais e nacionais de MFC, organizadas pelo Grupo de Trabalho de Mulheres na MFC, da SBMFC. Isso nos leva a questionar se os Programas de Residência em MFC no Brasil estão oferecendo as noções básicas de abordagem da 
mulher em situação de gravidez indesejada. É fundamental que uma discussão crítica e reflexiva sobre o aborto inseguro seja realizada nas escolas médicas e nos programas de residência, considerando que este é um problema de saúde pública relevante para a nossa sociedade.

\section{Da teoria à prática - A experiência do Fórum Aborto Legal RS}

Em Porto Alegre, um grupo específico para trabalhar na questão do aborto se constituiu a partir de uma iniciativa das Mulheres na Universidade e na Saúde (MUSAs), grupo de professoras da Universidade Federal do Rio Grande do Sul (UFRGS) constituído em 2015. No âmbito de um projeto de extensão para debater assuntos pouco falados na universidade, especialmente aqueles envolvendo questões de gênero, discriminação e injustiças sociais, as MUSAs organizaram rodas de conversa sobre o tema do aborto, que atraíram grande público, entre professoras(es), estudantes, profissionais de saúde e integrantes de organizações da sociedade civil.

Desses debates, surgiram ideias de ações concretas, daí a necessidade de formar um grupo operacional de trabalho para levá-las adiante. Assim, em julho de 2016, reuniu-se pela primeira vez o Grupo de Trabalho sobre Aborto Legal. Em 2017, o Grupo se transformou em Fórum, devido à sua crescente dimensão. Seus setores integrantes são: academia (MUSAs/UFRGS, incluindo professoras e estudantes), serviços de saúde (Hospital Materno Infantil Presidente Vargas), serviço de referência para atendimento de violência contra a mulher e aborto legal), Estado (Secretaria Estadual de Saúde do Rio Grande do Sul - Área de Saúde da Mulher) e sociedade civil (Marcha Mundial das Mulheres, Themis Gênero, Justiça e Direitos Humanos e Coletivo Feminino Plural). Recentemente, várias outras entidades se aproximaram do Fórum, como Sindicato dos Enfermeiros do Rio Grande do Sul, Conselho Regional de Psicologia, Conselho Municipal dos Direitos das Mulheres e Universidade Federal de Ciências da Saúde de Porto Alegre (UFCSPA).

O Fórum Aborto Legal RS, atualmente materializado em projeto de extensão na UFRGS, propõe-se a desenvolver ações concretas para tornar mais acessível e qualificar o atendimento às mulheres que buscam um aborto previsto em lei no RS. A discussão, de caráter interdisciplinar, acontece em diversas atividades: reuniões regulares (geralmente mensais); diálogo com gestores, serviços de saúde e entidades de classe profissional; organização dos Colóquios sobre Aborto Legal no RS; participação em eventos diversos, acadêmicos ou não; produção de materiais de divulgação; atividades de formação continuada, dentre outras.

Vale a pena destacar a realização das três edições do Colóquio sobre Aborto Legal no RS, a atividade mais emblemática realizada pelo Fórum. A primeira edição ocorreu em novembro de 2016 no Hospital Materno Infantil Presidente Vargas, no intuito de envolver centralmente este serviço, considerado a maior referência no RS para atendimento de violência sexual e aborto legal, no esforço pela qualificação dos serviços no Estado. A segunda edição, que destacou a importância do trabalho em rede, ocorreu em setembro de 2017 no Hospital de Clínicas de Porto Alegre, e discutiu-se sobre a importância da APS nesse contexto. O terceiro Colóquio aconteceu em 2018, nas dependências das duas universidades (UFRGS e 
UFCSPA), com o tema "Garantia do direito ao aborto legal por meio da educação", tendo como destaque as discussões sobre aborto legal no âmbito de ensino e formação.

Cada Colóquio originou uma série de encaminhamentos, que vêm sendo trabalhados no Fórum, tais como: realização de um monitoramento dos serviços de referência pela Secretaria Estadual de Saúde juntamente com o Ministério Público do RS; visitas aos serviços para dialogar sobre o funcionamento e as práticas realizadas; promoção de espaços de diálogo entre os profissionais que trabalham na linha de frente; propostas de inserção curricular de conteúdos envolvendo violência contra as mulheres e aborto legal nos cursos da área da saúde.

Outra atividade permanente do Fórum é a produção de materiais de divulgação, incluindo: um panfleto/cartaz informativo para o público em geral, contendo informações sobre os direitos relacionados ao atendimento de mulheres vítimas de violência sexual e ao aborto legal, indicando os serviços de referência no Estado e telefones úteis para informações adicionais; e um vídeo educativo intitulado "O aborto permitido por lei no Brasil”, realizado com entrevistas e depoimentos filmados na ocasião do primeiro Colóquio. $\mathrm{O}$ vídeo, desenvolvido em parceria com o Telessaúde RS, está disponível em acesso livre na internet.

O Fórum Aborto Legal RS está sendo uma experiência muito positiva, com resultados concretos nos processos de sensibilização e responsabilização com a questão do aborto legal. O envolvimento de diversos setores (academia, assistência, estado e movimento social) torna a discussão muito rica e aumenta a legitimidade do trabalho, pois as ações, no seu planejamento, passam pelos diferentes pontos de vista antes de serem postas em prática. Um aprendizado constante para todas que participam e um resultado certamente melhor para a sociedade.

\section{Considerações finais}

O Brasil, onde o contexto legal em relação à prática do aborto voluntário é dos mais restritivos do mundo, está indo na contramão das recomendações das autoridades internacionais de saúde e das evidências científicas. Como a OMS reitera em todo documento sobre o assunto, a descriminalização do aborto é uma estratégia fundamental para contemplar os direitos sexuais e reprodutivos e diminuir a mortalidade materna. ${ }^{30}$

Em conclusão, no nosso contexto de restrição legal, um primeiro passo para avançar no direito ao aborto é fazer cumprir a lei que garante a sua realização em situações específicas, como no caso da violência sexual, tão frequente no Brasil. Por fim, a atuação dos(as) profissionais da atenção primária na redução de danos parece ser uma estratégia chave para evitar abortos inseguros e, por consequência, reduzir a mortalidade materna.

\section{Conflito de interesses}

Camila Giugliani declara, em nome de todas as coautoras do manuscrito, que são integrantes do Grupo de Trabalho Mulheres na MFC da SBMFC e/ou do Fórum Aborto Legal RS e sustentam posição favorável à legalização do aborto, pelos argumentos expostos no artigo. 


\section{Contribuição dos autores}

CG foi responsável pela concepção e estrutura do artigo. Todas as autoras contribuíram igualmente na redação do artigo, revisaram e aprovaram as suas versões preliminar e final.

\section{Agradecimentos}

Agradecemos às integrantes do Fórum Aborto Legal RS pela partilha e pela construção de um belo projeto coletivo.

\section{Referências}

1. Kryiakos N, Fiorini E. A dimensão legal do aborto no Brasil. In: Aborto Legal: Implicações Éticas e Religiosas. São Paulo: Cadernos Católicas pelo Direito de Decidir; 2002. p. 131-46.

2. Fleury-Teixeira E, Meneghel SN. Dicionário Feminino da Infâmia: acolhimento e diagnóstico de mulheres em situação de violência. Rio de Janeiro: FIOCRUZ; 2015.

3. Silva BGC, Lima NP, Silva SG, Antúnez SF, Seerig LM, Restrepo-Méndez MC, et al. Mortalidade materna no Brasil no período de 2001 a 2012: tendência temporal e diferenças regionais. Rev Bras Epidemiol. 2016;19(3):484-93. http://dx.doi.org/10.1590/19805497201600030002

4. Victora CG, Aquino EML, Leal MC, Monteiro CA, Barros FC, Szwarcwald CL. Saúde de mães e crianças no Brasil: progressos e desafios Lancet. 2011;32-46. DOI: http://dx.doi.org/10.1016/S0140-6736(11)60138-4

5. Diniz D, Medeiros M, Madeiro A. Pesquisa Nacional de Aborto 2016. Ciênc Saúde Coletiva. 2017;22(2):653-60. http://dx.doi. org/10.1590/1413-81232017222.23812016

6. Madeiro AP, Diniz D. Serviços de aborto legal no Brasil - um estudo nacional. Ciênc Saúde Coletiva. 2016;21(2):563-72. DOI:0.1590/141381232015212.10352015

7. Fórum Brasileiro de Segurança Pública - FBSP. Anuário brasileiro de segurança pública. São Paulo: Edição VIII; 2014.

8. Cerqueira D, Coelho DSC. Estupro no Brasil: uma radiografia segundo os dados da saúde. Brasília: Ipea; 2014.

9. Brasil. Ministério da Saúde. Secretaria de Políticas de Saúde. Assistência Integral à Saúde da Mulher: Bases da Ação Programática. Brasília: Ministério da Saúde; 1984.

10. Cerqueira D, Coelho DSC, Ferreira H. Estupro no Brasil: vítimas, autores, fatores situacionais e evolução das notificações no sistema de saúde entre 2011 e 2014. Rio de Janeiro: IPEA; 2017.

11. Código Penal Brasileiro de 1940. Decreto-Lei No. 2.848 de 7 de dezembro de 1940. Brasília: Rio de Janeiro; 1940.

12. Brasil. Ministério da Saúde. Secretaria de Políticas de Saúde. Prevenção e tratamento dos agravos resultantes da violência sexual contra mulheres e adolescentes: norma técnica. Brasília: Ministério da Saúde; 1999.

13. Brasil. Ministério da Saúde. Portaria No 528 de 1 de abril de 2013. Define regras para a habilitação do funcionamento dos serviços de Atenção Integral às Pessoas em Situação de Violência Sexual no âmbito do Sistema Único de Saúde (SUS). Brasília: Ministério da Saúde; 2013.

14. Brasil. Ministério da Saúde. Secretaria de Atenção à Saúde. Departamento de Ações Programáticas Estratégicas. Área Técnica de Saúde da Mulher. Prevenção e Tratamento dos Agravos Resultantes da Violência Sexual contra Mulheres e Adolescentes: norma técnica. Brasília: Ministério da Saúde; 2012.

15. Talib R, Citeli MT. Serviços de Aborto Legal em hospitais Públicos brasileiros (1989-2004) Dossiê. São Paulo: Cadernos Católicas pelo Direito de Decidir; 2005.

16. Faúndes A, Duarte GA, Osis MJD, Andalaft Neto J. Variações no conhecimento e nas opiniões dos ginecologistas e obstetras brasileiros sobre o aborto legal, entre 2003 e 2005. Rev Bras Ginecol Obstet.2007;29(4):192-9. http://dx.doi.org/10.1590/S0100-72032007000400005

17. G1. Turma do STF abre precedente para descriminalizar aborto até terceiro mês [Internet]. [acesso 2018 Jun 27]. Disponível em: http:// g1.globo.com/politica/noticia/2016/11/turma-no-supremo-derruba-prisao-de-equipe-medica-de-clinica-de-aborto.html 
18. Expresso. O que diz a PEC 181. E qual seu impacto sobre o aborto legal [Internet]. [acesso 2018 Jun 27]. Disponível em: https://www. nexojornal.com.br/expresso/2017/11/10/O-que-diz-a-PEC-181.-E-qual-seu-impacto-sobre-o-aborto-legal

19. Theme-Filha MM, Baldisserotto ML, Fraga AC, Ayers S, da Gama SG, Leal MD. Factors associated with unintended pregnancy in Brazil: cross-sectional results from the Birth in Brazil National Survey, 2011/2012. Reprod Health. 2016;13(Suppl 3):118. http://dx.doi.org/10.1186/ s12978-016-0227-8

20.Zamberlin N, Romero M, Ramos S. Latin American women's experiences with medical abortion in settings where abortion is legally restricted. Reprod Health. 2012;9(1):34. http://dx.doi.org/10.1186/1742-4755-9-34

21. Briozzo L, Vidiella G, Rodríguez F, Gorgoroso M, Faúndes A, Pons JE. A risk reduction strategy to prevent maternal deaths associated with unsafe abortion. Int J Gynaecol Obstet. 2006;95(2):221-6. http://dx.doi.org/10.1016/j.ijgo.2006.07.013

22. Pan American Health Organization - PAHO. Changing relationships in the health care context: the Uruguayan model for reducing the risk and harm of unsafe abortions. Montevideo: Pan American Health Organization; 2012. $30 \mathrm{p}$.

23. Grossman D, Baum SE, Andjelic D, Tatum C, Torres G, Fuentes L, et al. A harm-reduction model of abortion counseling about misoprostol use in Peru with telephone and in-person follow-up: A cohort study. PLoS One. 2018;13(1):e0189195. http://dx.doi.org/10.1371/journal. pone.0189195

24. Matía MG, Trumper EC, Fures NO, Orchuela J. A replication of the Uruguayan model in the province of Buenos Aires, Argentina, as a public policy for reducing abortion-related maternal mortality. Int J Gynaecol Obstet. 2016;134(S1):S31-4. http://dx.doi.org/10.1016/j. ijgo.2016.06.008

25. Faúndes A. Abortamento. In: Duncan BB, Schimdt MI, Giugliani ER, Duncan MS, Giugliani C, eds. Medicina Ambulatorial: Condutas de Atenção Primária baseadas em evidências. 4ํe ed. Porto Alegre: Artmed; 2014.

26. Starfield B. Primary care: concept, evaluation, and policy. New York: Oxford University Press; 1992.

27. Brasil. Ministério da Saúde. secretaria de atenção à saúde. departamento de ações Programáticas estratégicas. Atenção humanizada ao aborto: norma técnica. $2^{a}$ ed. Brasília: Ministério da Saúde; 2011.

28. World Health Organization - WHO. Reproductive health strategy to accelerate progress towards the attainment of international development goals and targets. Geneva: World Health Organization; 2004.

29. Conselho Federal de Medicina. Código de Ética Médica. Resolução CFM no 1.931, de 17 de setembro de 2009. Brasília: Conselho Federal de Medicina; 2010.

30. Organização Mundial de Saúde - OMS. Abortamento seguro: orientação técnica e de políticas para sistemas de saúde. $2^{\text {a }}$ ed. Genebra: Organização Mundial de Saúde; 2013.

31. Rubin SE, Godfrey E, Gold M. Patient attitudes toward early abortion services in the family medicine clinic. J Am Board Fam Med. 2008;21(2):162-4. http://dx.doi.org/10.3122/jabfm.2008.02.070158

32. Fernandes KG, Camargo RP, Duarte GA, Faúndes A, Sousa MH, Maia Filho NL, et al. Knowledge of medical abortion among Brazilian medical students. Int J Gynaecol Obstet. 2012;118 Suppl 1:S10-4. http://dx.doi.org/10.1016/j.ijgo.2012.05.004

33. Lesnewski R, Prine L, Gold M. New research abortion training as an integral part of residency training. Fam Med. 2003;35(6):386-7.

34. Herbitter C, Bennett A, Schubert FD, Bennett IM, Gold M. Management of early pregnancy failure and induced abortion by family medicine educators. J Am Board Fam Med. 2013;26(6):751-8. http://dx.doi.org/10.3122/jabfm.2013.06.120248

35. Brahmi D, Dehlendorf C, Engel D, Grumbach K, Joffe C, Gold M. A descriptive analysis of abortion training in family medicine residency programs. Fam Med. 2007;39(6):399-403.

36. Sociedade Brasileira de Medicina de Família e Comunidade. Currículo baseado em competências para Medicina de Família e Comunidade, org. Lermen Junior N. Rio de Janeiro: Sociedade Brasileira de Medicina de Família e Comunidade; 2015. [acesso 2018 Jan 29]. Disponível em: https://www.ufrgs.br/telessauders/documentos/biblioteca_em_saude/006_material_saude_curriculo_competencias_mfc.pdf 